\title{
Medicina supresora y medicina estimulante: una mirada penetrante de la práctica médica
}

\author{
Leonardo Viniegra-Velázquez*
}

Unidad de Investigación en Medicina Basada en Evidencias, Unidad de Hemato-Oncología e Investigación, Hospital Infantil de México Federico Gómez, Ciudad de México, México

\begin{abstract}
Resumen
La teoría de la medicina supresora (MS) y la medicina estimulante (ME) destaca dos formas radicalmente distintas de entender la enfermedad y de enfrentarla. Para la MS es un objeto extraño o ajeno al organismo que hay que disminuir o destruir (suprimir); para la ME es un trastorno de la armonía interna que requiere fortalecer o avivar el organismo para restablecerla (estimular). En la medicina moderna, el poder de la industria de la salud favorece el predominio de la MS, por su alta rentabilidad, y la marginación de las medicinas alternativas (MA), poco rentables que, al igual que la vacunación o las terapias sustitutivas y regenerativas, son formas de ME. El efecto placebo (EP) inherente a la práctica médica, revelador de fuerzas curativas endógenas susceptibles de estimulación, da sentido a la ME y credibilidad a las MA. La dirección del EP de lo macro (psicosocial) a lo micro (físico-químico) explica su alta especificidad y ausencia de efectos secundarios. El efecto farmacológico de lo micro a lo macro, opuesto a las fuerzas endógenas, conlleva indefectiblemente efectos secundarios que requieren ulteriores supresiones y dosis repetidas indefinidamente. Se analizan equívocos de la ciencia con respecto al EP y al imponer a las MA criterios metódicos propios de la MS, que las desvirtúa, descalifica y excluye como objetos de conocimiento. Se insiste en la necesidad de reconocer la ME y rescatar las MA para la indagación, a fin de explorar sinergias, complementos o reemplazos con relación a la MS en la búsqueda del bien vivir.
\end{abstract}

Palabras clave: Medicina supresora. Medicina estimulante. Efecto placebo. Efecto farmacológico. Medicinas alternativas. Enfermedad crónica.

\section{Suppressive medicine and stimulant medicine: a penetrating view in medical practice}

\section{Abstract}

The theory of suppressive (SuM) and stimulant (StM) medicine highlights two radically different ways of understanding and coping with diseases. For SuM it is a strange or foreign object to the organism that must be diminished or destroyed (suppressed); for StM it is a disorder of internal harmony that requires strengthening or enlivening the body to re-establish it (stimulate). In modern medicine, the power in the health industry favors the predominance of SuM, because of its high profitability, and the marginalization of low-cost alternative medicines (AM) that, like vaccination or substitutive and regenerative therapies, are forms of StM. The placebo effect $(P E)$ inherent to medical practice, revealing of endogenous curative forces susceptible to stimulation, gives meaning to StM and credibility to AM. The direction of the PE from the macro (psychosocial) to the micro (physical-chemical) explains its high specificity and absence of side effects. The pharmacological

Correspondencia:

*Leonardo Viniegra-Velázquez

E-mail: leonardo.viniegra@gmail.com
Disponible en internet: $25-09-2018$ Bol Med Hosp Infant Mex. 2018:75:267-278 www.bmhim.com

1665-1146/@ 2018. Hospital Infantil de México Federico Gómez, impreso por Permanyer México SA de CV, todos los derechos reservados. 
effect of the micro to the macro, opposed to the endogenous forces, inevitably entails side effects that require further suppression and indefinite repetition of doses. Scientific assertions that misunderstand PE, and impose on the AM methodical criteria of the SuM that detract, disqualify and exclude them as objects of knowledge, are analyzed. The emphasis is on the need to recognize the StM and rescue the AM for inquiry in order to explore synergies, complements or replacements in relation to the SuM, in the quest for to live well.

Key words: Suppressive medicine. Stimulant medicine. Placebo effect. Pharmacological effect. Alternative medicines. Chronic disease.

Qué ciencia es esta que permanece indiferente, acomodaticia, aquiescente o cómplice de la dominancia de los intereses de lucro sin límite, que hunden la civilización y devastan el planeta...

El autor

\section{Introducción}

Desde sus albores, los humanos enfrentaron el sufrimiento físico y moral, el misterio y la inexorabilidad de la muerte. En el transcurrir, surgieron los iniciados, los encargados por la comunidad de ayudar a los sufrientes y mitigar sus malestares. También se fueron reconociendo patrones del padecer de las comunidades y construyendo descripciones y explicaciones de los males identificados. Desde etapas tempranas se hizo una distinción tajante entre las vivencias que anunciaban o implicaban diversos tipos de sufrimiento y las propias de una vida usual. Las primeras se pensaron esencialmente distintas de las segundas. Surgió, así, la noción de enfermedad (o sus equivalentes en diversas culturas), considerada contraria al deber ser del organismo ${ }^{1}$. En nuestro tiempo, la enfermedad es una vivencia, transitoria o permanente, intempestiva o previsible, repentina o paulatina, que se aparta del bienestar - lo que R. Leriche llamaba «la vida en el silencio de los órganos", citado por G. Canguilhem²-, como sufrimientos físicos o morales; impedimentos o limitaciones para llevar a cabo las tareas 0 actividades de la vida diaria; inconvenientes para la convivencia, para bastarse a sí mismo, para disfrutar de las buenas cosas de la existencia o la muerte prematura. Históricamente, las diversas culturas han configurado sus modos de enfermar, de expresión de enfermedades, de percibirlas y reconocerlas, de actuar sobre ellas, de sobrellevarlas y de morir'. En ese devenir histórico surgieron ideas ontológicas de las enfermedades (¿qué son?) con una variedad comprendida entre dos opuestos:

- El que predominó en el mundo occidental de raíz eurocéntrica las pensó como afección extraña o ajena a la naturaleza humana $y$, a la postre, como entidad u objeto independiente y autónomo del organismo (extrínseca). ${ }^{a}$

- El otro, prominente en Asia y en algunas culturas originarias, las consideró intrínsecas al organismo: trastorno de la armonía interna o de los flujos de energía vital, atribuido a discordancias o transgresiones del orden natural ${ }^{3}$.

En el desarrollo de las diversas doctrinas y prácticas médicas que aparecieron en la geografía planetaria, predominó uno u otro polo. El caso occidental donde «el objeto extraño y ajeno" dio lugar a estrategias de acción para disminuirlo o eliminarlo (anti-enfermedad), se denomina medicina supresora (MS) ${ }^{3}$. Las otras medicinas de asiento en el lejano oriente y en tradiciones ancestrales (medicinas alternativas [MA]), donde el objeto es la persona enferma y las estrategias de acción buscan fortalecer, avivar, armonizar o liberar el objeto a fin de restablecer su equilibrio y sintonía con el entorno, son representativas de la medicina estimulante (ME) ${ }^{3}$. La especificación, las implicaciones y la contrastación entre ambas medicinas es el motivo principal del presente ensayo.

\section{La medicina occidental y su desarrollo}

En la medicina occidental (MO) moderna, preponderante a escala global, la idea de enfermedad como afección extraña 0 ajena a la condición humana se asoció con la de salud (deber ser y contraparte de la enfermedad), y se integró el binomio salud-enfermedad, paradigma rector de los quehaceres de atención y cuidado de la salud que, entre otras cosas, requirió del desarrollo de infinidad de indicadores de «normalidad», construidos basándose en patrones estadísticos que, a manera de referentes, permitieran objetivar la presencia

a Paradójicamente, la medicina hipocrática considerada fundacional de la medicina occidental con la idea de enfermedad como desequilibrio humoral atribuido a la forma de ser y de vivir de las personas (rasgos temperamentales y hábitos), evidencia un claro predominio de la idea intrínseca de la enfermedad. 
de alteraciones o desviaciones y sustentar criterios diagnósticos. Ello implicó la reducción de la idea de enfermedad a la de desviación y el auge de la medicina tecnológica, con el desarrollo de diversas tecnologías, como las diagnósticas, para la cuantificación precisa y el registro de indicadores anatómicos, funcionales y moleculares, contrastarlos con los "patrones normales» y estimar el número y grado de desviaciones; y las terapéuticas, médicas o quirúrgicas, cada vez más potentes y específicas, tendientes a suprimir la enfermedad identificada o dosificar con precisión medidas correctivas de las desviaciones, a fin de aproximarlas a los patrones referenciales (terapia de control).

La MO universalizada encarna en instituciones públicas y privadas a todos los niveles: local, estatal, nacional e internacional, dedicadas al «cuidado de la salud humana" (el entrecomillado destaca que se trata de un señuelo manipulador, dado que sus actividades corresponden, stricto sensu, a la lucha contra las enfermedades, esos objetos extraños y ajenos que hay que eliminar). Los sistemas de salud operan bajo enfoques poblacionales 0 individuales, por medio de estrategias de acción con propósitos complementarios que, de acuerdo con la racionalidad nosológica de la historia natural de las enfermedades ${ }^{4}$, deben implementarse de manera secuencial: ${ }^{b}$

- La prevención con enfoque poblacional predominante y que, de acuerdo con el momento y oportunidad de su realización, se clasifica como primaria (desiderátum de la salud pública), a la que pretende retrasar o impedir la aparición de determinada enfermedad; secundaria, a la dirigida a detectarla en estadios tempranos con el propósito de retardar o detener su desarrollo; terciaria, cuya finalidad, una vez presente la enfermedad, es evitar que empeore o se produzcan complicaciones, agudizaciones o recaídas; cuaternaria, orientada a atenuar o evitar las consecuencias perjudiciales de las intervenciones innecesarias o excesivas del sistema sanitario ${ }^{5}$.

- El diagnóstico, como parte de las estrategias que se implementan a nivel individual, consiste en identificar factores presentes en los individuos que elevan la probabilidad de aparición de una enfermedad en un futuro próximo (enfoque de riesgo) o en identificar su

b La promoción de la salud que, según la carta de Ottawa, "consiste en proporcionar a la gente los medios necesarios para mejorar la salud y ejercer un mayor control sobre la misma", no forma parte propiamente de las estrategias formales de lucha contra las enfermedades y de las responsabilidades directas de las instituciones de salud. presencia en la persona que requiere o demanda la atención (enfoque de daño).

- El tratamiento, con una primera distinción entre lo médico (incluye trastornos somáticos o psicológicos) y lo quirúrgico. De acuerdo con sus fines y posibilidades, los tratamientos pueden ser: a) preventivos, cuando corresponden a la fase de prevención que intenta lentificar el desarrollo de una enfermedad y atenuar o evitar su agravamiento o complicaciones; b) curativos, dirigidos a atenuar 0 suprimir la enfermedad; c) sustitutivos de la deficiencia o pérdida de la función de algún órgano; d) de control, mantener las desviaciones de las constantes clínicas y metabólicas o de los estándares mentales del paciente dentro de rangos aceptables de acuerdo con criterios científicos o clínicos; e) paliativos, cuyo propósito es mitigar el sufrimiento o prolongar el lapso de vida en aceptables condiciones ante lo incurable o terminal; f) de rehabilitación, los cuales buscan restablecer la funcionalidad afectada o prevenir y atenuar secuelas que puede provocar la enfermedad en cuestión.

Este ideal de racionalidad del cuidado de la salud, difícil de objetar, entra en colisión con la lógica que impera en nuestro tiempo, donde el predominio abrumador de los intereses de lucro sin límite «aniquila los anhelos de libertad y justicia de los pueblos; arrasa los valores propios de convivencias respetuosas, solidarias, cooperativas y fraternas, y devasta el ecosistema planetario ". En el ámbito que nos ocupa, esos intereses están representados por la poderosa «industria de la salud", que lo ha configurado de acuerdo con las prioridades de ganancia y rentabilidad de los negocios, muy por encima de cualquier otra consideración. Así, la prevención, la estrategia de mayor trascendencia y beneficio potencial para la población, es de escaso interés para la industria porque su eficacia creciente implicaría una merma de consumidores y «malos tiempos» para los negocios. De ahí, las prioridades de inversión en innovaciones diagnósticas y terapéuticas en todas sus variantes. El poder de esta industria se patentiza en el operar de las instituciones que bombardean a la población con propaganda que la incita a responsabilizarse del cuidado de la propia salud y a frecuentar los servicios médicos, que ha alcanzado tal penetración en el imaginario social que configura cada vez más sus formas de ser y de vivir. La obsesión por la salud y el horror a la enfermedad se convierten en preocupación inextinguible que polariza la vida humana, con consumidores cada vez más dependientes de los servicios sanitarios, que incorporan, en sus modos 
de vivir, la racionalidad de la mirada médica: alerta perenne y lucha implacable contra las enfermedades, lo que se designa como medicalización de la vida, que aleja a las personas del "bien vivir».

La medicalización de legiones de consumidores «fabricados" por la industria (incluidos los prestadores de los servicios de salud) ${ }^{c}$ explica que los otrora factores de riesgo ahora sean enfermedades a combatir, y las vicisitudes de la existencia, que se afrontaban como peripecias menores o situaciones inherentes al diario vivir, se aprendía de ellas, se sobrellevaban y a menudo dejaban de representar inconvenientes serios (por ejemplo, los achaques con el avance de la edad que pueden limitar ciertas actividades físicas, pero propiciar otras de orden intelectual o artístico), ahora se convierten en "problemas de salud» que deben ser estudiados y tratados, lo que hace que las víctimas dependan de profesionales, y se sometan a estudios diagnósticos que, a menudo, derivan en sobrediagnósticos y terapias innecesarias ${ }^{7,8}$. También explica que «lo raro, pero normal» de otros tiempos, amerite intervenciones expertas, lo cual, además de un jugoso negocio de la industria, propicia que las instituciones refuercen su papel en el control social al hacer a los consumidores más dependientes, desviando su atención de los procesos sociales que subyacen a la presencia creciente y precoz de enfermedades (crónicas) somáticas y psíquicas: condiciones y circunstancias cada vez más adversas para formas de vida digna, estimulante, productiva, gratificante, serena y fraternal ${ }^{6}$.

\section{Hacia una mirada penetrante de la práctica médica}

A propósito del panorama actual descrito de la MO, se presenta una forma penetrante de mirar la práctica médica a través de las «lentes» que aportan los conceptos de medicina supresora (MS), que se refiere a la forma de proceder ante la enfermedad como afección extraña o ajena al organismo, y de medicina estimulante (ME), alusiva a los propósitos terapéuticos de fortalecer al organismo afectado por un trastorno de la armonía interna o de los flujos de energía vital ${ }^{3}$. Aunque tal dicotomía adolece de simplificación y

c En palabras de Zygmunt Bauman: «Los miembros de la sociedad de consumidores son ellos mismos unos bienes, y es la cualidad de ser un bien de consumo lo que los convierte en miembros de derecho de esa sociedad. Convertirse y seguir siendo un bien vendible es el objetivo máximo del consumidor, aunque en general sea de manera latente y pocas veces consciente». esquematismo, abre nuevos espacios de reflexión y entendimiento del quehacer social más influyente en la vida de las personas en una época donde suele imperar un pensamiento único y excluyente. Ambas, la MS y la ME (que no existen en "estado puro» sino en forma de predominios, porque desde tiempo atrás ocurrieron desviaciones, contaminaciones e hibridizaciones), revelan, a través de las ideas de enfermedad que las sustentan y las formas de proceder ante ellas, la coexistencia, aún hoy día, de formas tan divergentes de entender la vida humana.

Al confrontar la MS y la ME, no puede dejarse de lado que, en nuestro tiempo, la dominancia de los intereses de lucro sin límite que colapsan la civilización es el hilo conductor para explicar el predominio de unas ideas y prácticas sobre otras en los diversos campos del quehacer social, que no obedece a una supuesta superioridad, sino a su sintonía directa o indirecta con esos intereses. En el campo de la salud, el imperio de la idea de enfermedad como afección extraña o ajena, y su complemento lógico, la MS, dirigida a combatirla y eliminarla por todos los medios, representa una de las vetas más rentables por el creciente mercado de consumidores (medicalización). Además, es la cara visible de la quiebra civilizatoria en este campo al contribuir a la mercantilización de la vida: el precio de preservar la salud al alza y la dignidad prescindible; al impulsar la «deshumanización» de la medicina: la tecnificación que desplaza y minimiza el intercambio afectivo y cognoscitivo médico-paciente, e intensificar la medicalización que controla mentes y cuerpos, e infunde angustia, temor y desasosiego. En contraste, la idea de enfermedad como trastorno de la armonía interna y la ME orientada a restablecerla, con requerimientos tecnológicos modestos que implica, por parte de los pacientes, recuperar autonomía y, por tanto, menor dependencia y vulnerabilidad a la medicalización (simientes de una vida digna, mesurada y serena), al significar "consumidores apáticos o renuentes" para el gran negocio de la salud, representa un serio inconveniente, y la razón de fondo de que el carácter estimulante de la medicina haya sido ignorado, negado, soslayado y hasta vilipendiado, aunque está presente bajo muchos ropajes en la $\mathrm{MO}$ y en la gran mayoría de variantes que se agrupan bajo el calificativo despectivo de "medicinas alternativas no científicas».

Mirar la MO diferenciando el carácter supresor del estimulante, nos hace conscientes de que un gran logro civilizatorio, el incremento espectacular de la expectativa vital promedio (no aplicable a los países 
pobres), se debe a la prevención de enfermedades trasmisibles en virtud de la vacunación universal que corresponde a la ME, y al saneamiento ambiental propio de la promoción de la salud, cuyo propósito es anticipar el riesgo que representa la profusión y cercanía de fuentes infecciosas y contaminantes. También permite concienciar respecto a estrategias terapéuticas desarrolladas a propósito de enfermedades no trasmisibles (crónicas) en etapas tardías o terminales: la sustitutiva (funcional u orgánica), en especial el trasplante de órganos, corresponde igualmente con la ME, aunque la inmunosupresión se hace necesaria para evitar el rechazo hasta donde sea posible, lo que ocasiona la típica enfermedad iatrogénica del trasplante; la regenerativa (de tejidos y órganos), que entraña beneficios potenciales inimaginables en otros tiempos y se vislumbra como de lo más promisorio también forma parte de la ME. En ambos casos, su auge y presencia creciente sugiere la principal razón de su viabilidad: "contribuir a los buenos negocios de la industria», lo que conlleva el pesado e ineludible lastre del alto costo de las tecnologías implicadas y las limitaciones o imposibilidades para que la mayoría de la población blanco se beneficie.

\section{La medicina supresora}

La MS como estrategia terapéutica (médica o quirúrgica) contra las enfermedades no trasmisibles (crónico-degenerativas): cardiovasculares, respiratorias, digestivas, neurológicas, articulares o los cánceres, tiene exclusividad dentro de la MO. Esta forma de pensar la enfermedad y de enfrentarla permanece inconmovible a pesar de incontables fracasos, de efectos colaterales indefectibles 0 de notorias consecuencias contraproducentes al grado de motivar el desarrollo de la prevención cuaternaria 5 . La regla en todo esto es que la primera inicie una cadena de supresiones para contrarrestar efectos indeseables de la precedente; la supresión, al aplastar o silenciar reacciones orgánicas, con frecuencia las exacerba, lo que suele manifestarse como reactivación o agravamiento del problema, que obliga a mayor dosis o a fármacos más potentes para "controlar» con nuevos efectos secundarios que exigen otros tantos tratamientos supresores, reiniciando la cadena y cerrando el círculo vicioso. La enfermedad como objeto extraño y ajeno y la lucha por erradicarla como complemento, al operar como pensamiento único de las afecciones crónicas, ${ }^{d}$ favorece que se «normalicen» y soslayen sus graves limitaciones y su cauda de efectos indeseables, en parte por la convicción (ilusoria) de que, más temprano que tarde, se contará con la tecnología apropiada para eliminarlas. Tal fe impide ver que la lógica profunda al desarrollo tecnológico es responder primariamente a los intereses de lucro y operar principalmente como dispositivo de control de conciencias y cuerpos al servicio de la dominación. De ahí que lo más dinámico y prioritario sea lo militar, lo espacial, lo informático, lo médico o las tecnologías de información y comunicación $(\mathrm{TIC})^{9}$, sin soslayar que el costo de cada novedad supone excluir de sus beneficios potenciales a sectores de la población aún mayores.

Algunos éxitos de la MS, como "la lucha contra el cáncer» 0 «el control» de ciertas enfermedades (hipertensión arterial, hiperlipidemias, obesidad), dependen más de los afectados, en alerta y sospecha perennes, que son claves de un tratamiento oportuno, 0 en disposición para lograr cambios efectivos y saludables en sus hábitos de vida, que de la eficacia del tratamiento per se. En otros desarrollos promisorios, como las vacunas contra ciertos tipos de cáncer o los autotrasplantes de médula ósea para subsanar los estragos de la quimioterapia, es la ME la que está en juego.

Con respecto a las enfermedades infecciosas agudas, existen dos modalidades contrastantes: las de curso autolimitado que se «resuelven solas", que no requieren de supresores cuyos efectos secundarios pueden retardar 0 complicar la recuperación y que, salvo en caso de epidemias o complicaciones, solo ameritan «medidas caseras»; y las que suelen requerir fármacos, en las que el uso de antibióticos 0 antivirales no corresponde estrictamente a la MS, porque sus efectos buscan eliminar la infección y no suprimir directamente las reacciones orgánicas. El uso de supresores sintomáticos en ambas modalidades obedece a necesidades inducidas por la medicalización y a exigencias del mercado de trabajo: reincorporación pronta y expedita al trabajo para no interrumpir la explotación ni mermar las ganancias. En cuanto a la cirugía (que implica mutilación y es típica de la MS) es imprescindible en ciertos problemas agudos y graves cuando es oportuna y bien indicada, y una alternativa necesaria en problemas crónicos agudizados, complicados o incapacitantes que

d Por tal tipo de pensamiento el carácter supresor del tratamiento -una idea propuesta por el autor para captar las diferencias con aproximaciones terapéuticas propias de la medicina estimulante, que no es del dominio público- escapa a la conciencia, a la percepción y al interés cognitivo de los protagonistas. 
ameritan extirpaciones de tejidos u órganos dañados y reconstrucciones, injertos o prótesis.

\section{La medicina estimulante}

En el campo diversificado de las MA que da forma a la práctica médica no convencional, la enfermedad se piensa, con matices, como expresión de un trastorno de la armonía interna o de los flujos de energía vital del organismo, atribuido a hábitos y conductas que implican discordancias o transgresiones del orden natural. Tal práctica médica es emblemática de la ME, ${ }^{\mathrm{e}}$ donde las estrategias buscan restablecer la armonía interna o el bien fluir de la energía vital en sintonía con el entorno. Para la poderosa industria de la "salud», que ha condicionado y reducido el papel de la ciencia al de insumo clave de las innovaciones tecnológicas y mediatizado a los científicos apresados en el reduccionismo ${ }^{10}$, las MA son un obstáculo al gran negocio. De ahí la denuncia, a través de la ciencia, de las falacias de la MA y su carencia de bases científicas, y la recomendación de excluirlas de la práctica clínica ${ }^{11,12}$-en especial la homeopatía-, contribuyendo a la imagen negativa que se fomenta.

En la ME, el tratamiento no busca influencias circunscritas, sino difusas, sistémicas e individualizadas, porque su base es entender cada individualidad; es decir, tratar pacientes y no enfermedades no es retórica vacía porque lo que importa es penetrar en cada individualidad irreductible para elegir y aplicar «estímulos individualizados» al caso en cuestión (aunque no necesariamente lo logre). Tales principios y propósitos chocan con los criterios de cientificidad dominantes: «los únicos objetos de conocimiento válidos son colectividades agrupadas por la misma enfermedad, expuestas a un mismo tratamiento y, al diferenciar entre los grupos estudiados, lo relevante son los valores significativos de $p$ (el ilusorio deslinde y control de lo explicable por azar)". De aquí deriva la casi imposibilidad de las MA de proveerse de pruebas y argumentos científicos de efectividad terapéutica, porque en tales montajes, la individualidad es anulada y la individualización negada; es decir, para tener credibilidad se les obliga a desvirtuarse. En esto radica lo central de las objeciones de la ciencia oficial; empero, una ciencia que, por principio, excluye observaciones minuciosas de casos únicos

e La ME es una teoría del autor para captar las diferencias cualitativas entre la práctica médica dominante (supresora) y las no convencionales. Las MA son, según esto, formas y variantes de ME. potencialmente relevantes al conocimiento y soslaya la evidencia histórica de que cada enfermedad nueva del catálogo nosológico derivó de reconocer «anomalías a lo esperado" en virtud del estudio profundo del caso en cuestión, debe ser cuestionada y revisada porque desecha sabiduría práctica que ha resistido la prueba del tiempo (¿todas las generaciones son crédulas, incautas o torpes?); se arroga la verdad exclusiva y legítima y el derecho a combatir "disidencia y herejía»; suplanta la complejidad inherente al organismo, reduciéndolo a una «máquina perfecta» (mecanicismo), oscureciendo su entendimiento; da crédito solamente a los hechos y desestima las ideas (empirismo reduccionista), favorece un pensamiento teórico único, y descalifica los frutos del ingenio que nos hizo humanos.

\section{El efecto placebo}

La ME, que podría parecer fantasiosa e ilusoria a muchos fieles de la ortodoxia (supresora), tiene un fundamento empírico incontrovertible en la omnipresencia del efecto placebo (EP) en situaciones curativas de cualquier índole. El EP es el mejoramiento de síntomas, signos, malestares $u$ otros indicadores alterados del paciente, no atribuible a una intervención terapéutica (farmacológica, mecánica, quirúrgica o de otro tipo ${ }^{13}$. Tal efecto es resultado de una movilización sistémica específica, inducida por autosugestión, derivada de la confianza de mejoría depositada en ciertos objetos significativos del entorno: una médica afamad, un médico afectuoso, un sitio confortante, una inyección poderosa, una pastilla roja, un chamán reconocido, una imagen venerada o un lugar de culto $^{13}$, casi siempre por vivencias precedentes propias o indirectas (nótese que los objetos no se restringen a situaciones curativas formales - práctica médica- porque el EP es emblemático de la reacción universal al dolor humano que se presenta en circunstancias muy diversas, a condición de que la búsqueda y expectativa de alivio sea central). En otras palabras, el EP hace evidente la presencia de poderes curativos endógenos, cuya movilización anticipa o reproduce «lo esperado» (alivio de las molestias $)^{13}$ que, además, son susceptibles de ser estimulados, lo que da sentido a la teoría de la ME.

La presencia del EP en los más diversos montajes clínicos controlados se hizo patente desde tiempo atrás, cuando aún despertaba interés y búsqueda deliberada. Algunos ejemplos se mencionan a continuación: en diversos tipos de dolor (postraumático, posoperatorio, isquémico, inflamatorio, espasmódico o vascular ${ }^{14-19}$; remisión de la enuresis, la depresión o el insomnio ${ }^{20}$; 
mejoría del estado asmático, el temblor esencial o diversas dermatosis ${ }^{21}$; control del síndrome de deprivación en drogadictos ${ }^{22}$; elevación de la tensión arterial en el choque séptico o descenso en la hipertensión arterial ${ }^{23,24}$; cambios en niveles de acidez gástrica 0 de glucemia ${ }^{25,26}$; a lo que cabe agregar datos recientes acerca de mecanismos moleculares de las respuestas placebo en la analgesia, con participación de sistemas opioides, dopaminérgicos y de endocannabinoides ${ }^{27,28}$, reveladores de que el EP, si bien es una percepción subjetiva, se acompaña de diversas manifestaciones objetivas. Al respecto de esto último, describir cada vez con mayor detalle el EP en términos físico-químicos puede aportar a su comprensión; plantear lo físico-químico como la explicación del EP — de estirpe biológica- oscurece su entendimiento (reduccionismo).

El EP como forma objetivada de lo que los antiguos llamaban la vis medicatrix naturae (el poder sanador de la naturaleza), o sea las fuerzas curativas endógenas (neutralizadas en la MS) que permanecen latente mientras no sean incitadas por ciertas situaciones y circunstancias, capaces de aliviar males muy diversos. ${ }^{\dagger}$ Esta universalidad del EP, como ya se mencionó, es un fundamento empírico incontestable de la ME y de la potencialidad curativa de las MA, lo cual, por supuesto, no es garantía de que necesariamente logren lo que se proponen ni que sean inmunes al engaño $o$ al fraude, signos de nuestro tiempo.

Ahora, después de especificar el EP, es oportuno referirse a otros equívocos del pensamiento científico estrecho que confunde EP con "cosa placebo», y al malentendido de creer que «la cosa placebo» es la fuente única de EP, lo que impide percibir su presencia en tratamientos a base de fármacos. Estas confusiones tienen justificaciones prácticas y metodológicas: dan pie a la convicción (ilusoria) de que el EP puede investigarse, controlarse y medirse con plena objetividad y consistencia: si solo utilizo sustancias inertes, puedo atribuir lo observable al EP. En los montajes de algunos ensayos clínicos controlados, esa convicción hace creer que comparar un fármaco con una sustancia inerte permite separar y distinguir con nitidez el efecto farmacológico del $E P$, ignorando que, en cualquier situación curativa, suele presentarse el EP, cumpliendo expectativas independientemente de si se trata de un fármaco, un procedimiento, una maniobra o una sustancia inerte; a lo que se agrega que la confianza de mejoría en objetos

f No es especular en el vacío sugerir que una expresión mayúscula e inusual del EP es el trasfondo de la mayoría de "las curaciones imposibles y los milagros comprobados» del voluminoso anecdotario. significativos del entorno puede variar (por vivencias precedentes), y las expectativas de alivio cambiar con rapidez: fuertes, débiles, nulas o hasta de malestar que podrían inducir, en este último caso, el llamado efecto nocebo (EN) que se manifiesta en empeoramiento. Considerando esta polaridad de beneficio y perjuicio del EP y el EN, puede comprenderse la gran variabilidad potencial de expectativas presentes al inicio de una acción terapéutica, que explica por qué el EP es tan cambiante, puede fortalecerse 0 debilitarse en breve tiempo como resultado de asociar nuevas vivencias, más o menos favorables o desfavorables, que susciten expectativas de alivio fuertes, débiles 0 aun de malestar, inductoras de un EP fortalecido, disminuido o del EN. Se entienden así varias cuestiones que la medicina actual ni siquiera se plantea: que la mejoría atribuida a un medicamento pueda deberse más al EP que al farmacológico, que las vivencias iniciales de un tratamiento (alivio, nulidad de efecto o empeoramiento) puedan influir decisivamente en la resultante ulterior por el tipo de expectativas suscitadas (alivio o empeoramiento) que sumen o resten al efecto farmacológico, y que no puede descartarse el EN ante resultados pobres, indeseables o contraproducentes de ciertas terapias supresoras (quimioterapia, inmunosupresión). Lo anterior permite enjuiciar conclusiones sesgadas de la ciencia oficial al respecto como «el remedio $X$ no mostró diferencias significativas con el placebo y, por tanto, se recomienda su exclusión de la práctica clínica", que es injustificada, estrecha y reveladora de un pobre entendimiento del organismo, porque el EP es intrínseco, y a menudo puede significar mejoría sustancial, incluso superior a la de un fármaco. Además, el EP no implica efectos secundarios indeseables (el EN, que también es intrínseco, no debe confundirse con los efectos colaterales perjudiciales propios del uso de fármacos que, en tal caso, sí suelen ser incitadores del EN).

La dualidad EP y EN, latente en toda situación curativa, es reveladora y sugestiva de una propiedad vital más general y primaria que los incluye (la vis medicatrix naturae solo da cuenta del EP): la anticipación, ${ }^{9}$ movilización sistémica que se adelanta a las situaciones de interacción inminentes con objetos significativos, es la

g La anticipación es la movilización sistémica incesante y creativa de los seres vivos, de extraordinaria especificidad y selectividad, que se adelanta a las situaciones de interacción inminentes con los objetos significativos del entorno (en virtud de la interiorización precedente de los vínculos con tales objetos). La anticipación explica el cambio perpetuo y la progresividad hacia la maduración que caracterizan el proceso vital individual en la escala temporal de la ontogenia, y hacia la diversificación y complejidad creciente de poblaciones de especies en la escala temporal de la filogenia. 
cualidad vital por excelencia ${ }^{29}$. La anticipación explica que, en un caso, las expectativas de mejoría inciten el EP y que, en el otro, las desfavorables, el EN. A propósito del poder explicativo de la anticipación, cabe referirse a experimentos de comportamiento condicionado reveladores de movilizaciones sistémicas anticipatorias que asocian estímulos condicionados (EC) y estímulos no condicionados (ENC) simultáneos en varias sesiones consecutivas, al término de las cuales el solo EC reproduce los efectos del ENC. Dos ejemplos, uno de inmunosupresión en ratas, que asoció ciclofosfamida intraperitoneal (ENC) con sacarina oral (EC). Después de varias sesiones, se observó que el EC por sí solo (sin el ENC, ya que solo se inyectó el solvente), reproducía (anticipaba) la inmunosupresión ${ }^{30}$. Otro añejo sobre hipoglicemia condicionada en enfermos psiquiátricos cuando aún se utilizaban los «choques insulínicos», después de series de crisis hipoglicémicas provocadas (ENC), la inyección exclusiva del vehículo sin la hormona en el sitio habitual (EC), reproducía la respuesta hipoglicémica ${ }^{31}$. ${ }^{\text {h }}$

Esto ilustra la cualidad de la anticipación: fuerza vital capaz de adelantarse a lo inminente y reproducir procesos aparatosos de gran complejidad (que se piensan privativos de ciertos fármacos) y especificidad que, en un sentido, revelan efectos impensados que suelen acompañar a la MS y, en otro, dan pie a vislumbrar alcances insospechados de estrategias de ME eficaces para amplificar el EP, lo cual abona a la credibilidad de las MA, hoy marginadas y descalificadas por el pensamiento único que impone una industria que progresa al crecer la morbilidad y lucra con la obsesión «enfermiza» por la salud, la fobia a la enfermedad y el terror a la muerte.

\section{El efecto farmacológico y el efecto placebo}

El efecto farmacológico (EF) se origina en el microcosmos físico-químico, al modificar interacciones moleculares a las que se atribuye «una contribución causal significativa» de la afección a tratar. Esta idea causal de base química aunada a la de tratamiento apropiado con fármacos (químicos), ejemplifica el

h El caso de las ratas no corresponde al EN. Es la anticipación que reproduce el intricado proceso de inmunosupresión en ausencia del fármaco provocador. En el caso de los «humanos torturados", las expectativas negativas de vivencias precedentes sí están en juego; de ahí que podría considerarse una forma de EN, donde la anticipación reproduce la crisis hipoglucémica en ausencia de insulina. pensamiento reduccionista que afirma: «el todo no es más que la suma de las partes» 0 «un sistema complejo solo puede explicarse por reducción a sus partes fundamentales»"32, donde el orden biológico (lo relativo al proceso vital en su complejidad irreductible donde tienen sentido los conceptos de anticipación, EP y EN) es una ficción. La diana de un fármaco es un elemento infinitesimal dentro de una inmensa diversidad integrada y jerarquizada: segmento mínimo de una secuencia de reacciones que, a su vez, es eslabón de cadenas, que integran redes, que dan forma a mecanismos intrincados que se articulan y acoplan dentro de caminos metabólicos y funcionales bajo los imperativos de la regulación y la integración orgánicas que hacen posible el carácter sistémico, cíclico y rítmico del proceso vital de un organismo en interacción incesante con los objetos significativos de su entorno (la complejidad del orden biológico irreductible al físico-químico). Así, la introducción de una sustancia ajena y reactiva en tal "concierto vital» de complejidad apenas imaginable, buscando el EF, da lugar a una suerte de "disarmonía» al modificar un segmento minúsculo cuya actividad o inactividad, en condiciones reales, depende de secuencias de reacciones de las que es parte, al interior de redes, de mecanismos, de ciclos, de ritmos, bajo imperativos sistémicos de una totalidad integrada e interactuante. Si consideramos que el EP resulta de una movilización de fuerzas curativas endógenas en sentido inverso al EF (de lo macro psicosocial a lo micro físico-químico), se comprende que esa disarmonía implique oposición a tales fuerzas, que las debilite o distorsione, expresándose en la presencia indefectible de "efectos secundarios" (impredecibles, desconocidos), y en la necesidad de repetir indefinidamente el fármaco reproductor de la modificación química disruptiva, que mantiene un EF con tendencia a debilitarse por las fuerzas endógenas opuestas, y requiere incremento de la dosis, con lo que acrecienta el riesgo de aparición de nuevos o más graves efectos secundarios. He aquí la justificación de la teoría de la MS.

El EP se origina por interacciones en la esfera psicosocial, es expresión de la anticipación y consiste en una movilización sistémica de gran especificidad, inducida por la confianza de mejoría y guiada por expectativas de alivio, que se adelanta a lo esperado y se manifiesta en mejoramiento de trastornos de lo más diverso. A primera vista, parecería que el EP es una reproducción fiel del EF, lo que haría suponer que es provocado por modificaciones físico-químicas análogas. Sin embargo, existe una diferencia radical: el EP obedece a un patrón de movilización sistémica anticipatoria (no a una 
modificación directa de interacciones químicas buscando ilusoriamente efectos circunscritos), que nace en la esfera psicosocial (cúspide de la complejidad) y sigue trayectos a través de los diferentes niveles de organización, que provoca mejoría con una especificidad que no deja de asombrar, no asociada a efectos secundarios. Se trata de una movilización sistémica inducida que, a través de niveles descendentes de complejidad, provoca reacomodos (armónicos) más o menos estables en pautas de comportamiento, flujos funcionales y metabólicos, mecanismos o cadenas de reacciones que anticipan lo esperado. Al respecto, es paradójico que una modificación minúscula del microcosmos molecular buscando efectos muy circunscritos (EF) implique distanciarse de la especificidad (efectos secundarios), y que el EP inducido en la esfera psicosocial sea realmente específico (ausencia de efectos secundarios). Dicho de otra manera, en la especificidad de efectos, la clave no está en la composición química del remedio (visión reduccionista) que provoca disarmonía (disrupción), sino en la cualidad de la movilización sistémica anticipatoria (armónica) que es dirigida por las expectativas.

\section{Diagnóstico y tratamiento en la medicina supresora y la medicina estimulante}

Ahora, cabe precisar otras diferencias entre la MS y la ME respecto al diagnóstico y el tratamiento. En la MS, aunque declare la primacía del enfermo sobre la enfermedad, diagnosticar es clasificar al paciente según el objeto (extraño y ajeno) identificado, que lo equipara con el resto de la población afectada, y la terapia es, con variantes, la misma para todos. Así, la individualidad se anula y el ideal de individualización terapéutica se diluye. En la ME, «el diagnóstico» es caracterizar la individualidad que sufre y el tratamiento individualizado busca movilizar las fuerzas curativas endógenas a fin de restablecer la armonía. Si hay acuerdo en que la curación (de una afección crónica) es una situación existencial libre o atenuada -pero estable- de la afección, carente de efectos secundarios que, cuando llega a ocurrir, permanece

i En la nosología oficial, las enfermedades son modelos teórico-estadísticos acerca de complejos o agrupamientos sindromáticos o paraclínicos, con referencia a la etiología o patogenia, que configuran sus propios criterios de validez interna y de constatación empírica, a través de la cuantificación de desviaciones (del patrón de normalidad) de indicadores biológicos: anatómico-funcionales-metabólicos-moleculares, de gráficas o de imágenes (este concepto alude principalmente a las enfermedades crónico-degenerativas). y no revierte al corto plazo, es claro que se trata de una posibilidad privativa de la ME porque solamente puede ser el resultado de incitar el poder sanador del organismo (especificidad). En contraste, la MS no cura. Sea o no eficaz, se asocia con efectos secundarios más o menos adversos, y la brevedad del EF y su debilitamiento requiere de reiteradas o nuevas supresiones, que agregan más efectos secundarios, y así sucesivamente. De tal forma, el balance global de beneficios y perjuicios es, a menudo, desfavorable o hasta contraproducente.

\section{Medicina estimulante y medicinas alternativas}

La ME que encuentra en el EP su fundamento tiene su expresión más conspicua en la psicoterapia -no incluida dentro de las MA-, pero no así la asociada con fármacos, que es supresora. La psicoterapia implica interacciones en la esfera psicosocial por medio del lenguaje verbal y no verbal, que promueven (estimulan) movilizaciones sistémicas buscando rearreglos asociativos que remuevan afectividades fijadas en ciertos objetos, a fin de modificar o atenuar rasgos de comportamiento neurótico motivo de sufrimiento, o incitar estados serenos que atenúen malestares diversos, incluidos los orgánicos. En la psicoterapia, como en cualquier situación curativa, está implicado el EP -y rara vez el EN- según el tipo de expectativas precedentes o suscitadas en las sesiones iniciales, matizando los resultados subsiguientes. La práctica clínica clásica -en vías de extinción- supone, en parte, una forma de psicoterapia breve que, en ocasiones, puede ser decisiva en la mejoría (sin poder discernir su grado de participación porque se trata de un problema teórico). Con la medicina tecnológica, donde el intercambio médico-paciente tiende a lo mínimo, esta forma de psicoterapia per se y el EP asociado participan cada vez menos en los resultados del tratamiento, seguramente para mal.

Las MA concuerdan, con muchos matices, en la forma de entender la enfermedad y en sus estrategias terapéuticas: la enfermedad como trastorno de la armonía interna o de los flujos de energía vital del propio organismo, atribuido a discordancias con el orden natural, y el tratamiento orientado a restablecer la armonía o el bien fluir energético. Aquí un recuento simplificado de $\mathrm{MA}^{33}$ : la herbolaria, la acupuntura, la medicina ayurvédica, la homeopatía, la medicina energética, la medicina neuropática, la medicina ambiental, la hipnoterapia, la magnetoterapia, la terapia floral, la terapia de sonidos, la terapia de desintoxicación, la terapia enzimática, la 
terapia ortomolecular o el autocuidado del Qigong. En seguida, se incluye una breve descripción interpretativa de las clásicas ${ }^{33}$ (huelga decir que en estas situaciones curativas está presente el EP y rara vez el EN):

- La herbolaria, con saberes acumulados por milenios y acervos de centenas de miles de plantas de las que se han "estudiado científicamente» solo unas 5,000. Las hierbas medicinales se utilizan en forma de tisanas, extractos, tinturas, aceites esenciales, tabletas, ungüentos, pomadas o bálsamos ${ }^{33}$. Comúnmente se piensa que interactúan con el organismo en el plano físico-químico, lo cual requiere una precisión obligada. En el caso de hierbas medicinales reducidas a fármacos, que implica su desnaturalización, en el proceso que las descompone para aislar, depurar, concentrar y hacer biodisponibles los supuestos "principios activos", efectivamente las interacciones son a nivel físico-químico (lo biológico reducido a lo químico); en cambio, al usarlas en las formas tradicionales, se preserva su composición original (multiplicidad y proporción de sustancias) y su «carácter biológico", y las interacciones se dan en el mismo plano organizativo del organismo. Esto viene a cuento, porque a partir de las observaciones minuciosas y exhaustivas de S. Hanhemann (padre de la homeopatía), y otras innumerables hechas con posterioridad, resumidas en el aforismo «el veneno está en la dosis», toda esta copiosa evidencia acumulada (que desestima la ciencia oficial interesada en probar fármacos) es muy sugestiva de que las pequeñas dosis de los componentes, tal como se encuentran en una tisana (conjunción proporcionada de numerosos compuestos), tienen efectos estimulantes de las fuerzas curativas endógenas (ME) y que, por contraste, las dosis farmacológicas (altas concentraciones del "principio activo", donde la hierba ha sido desnaturalizada y reducida) suprimen esas fuerzas (MS). De ahí que las pruebas científicas de eficacia de las hierbas medicinales reducidas a fármacos (ensayo clínico controlado) signifiquen, según esto, «desvirtuar su naturaleza", y suplantar o desechar una inmensa sabiduría práctica de origen ancestral, con el añadido de que para la ME, la enfermedad (crónica), no es un objeto extraño y ajeno al organismo que debe eliminarse, sino la expresión de un trastorno sistémico (disarmonía) en una individualidad que requiere un tratamiento individualizado orientado a fortalecerla a fin de restablecer la armonía.

- La acupuntura, componente prominente de la milenaria medicina tradicional china, donde el conocimiento profundo y detallado de cada paciente es clave, en especial el estado de los flujos de energía detectado a través de los diferentes tipos de pulso, reveladores del estado de los meridianos corporales (especie de conductos donde circula la energía vital) y de sus respectivos órganos internos. Consiste en estimular puntos de la superficie corporal (existen varios centenares) con propiedades electromagnéticas sui generis (mayor conductancia eléctrica), que son vía de acceso a esos meridianos, con la pretensión de armonizar los flujos energéticos, y provocar una movilización sistémica de alta especificidad a fin de restablecer el funcionamiento orgánico alterado.

- La medicina ayurvédica, que data también de varios milenios, es un sistema médico que da importancia semejante al cuerpo, a la mente y al espíritu en el conocimiento del paciente (diagnóstico) que es, a su vez, referente de las estrategias para restablecer la armonía trastocada. Es el sistema de mayor integralidad, ya que combina diversas terapias naturales: dietas, ejercicios, meditación, hierbas, masajes o ejercicios respiratorios, y con base en enfoques diagnósticos altamente personalizados, combina y dosifica las terapias al diseñar esquemas de tratamiento individualizado.

- La homeopatía, medicina de origen occidental (finales del siglo XVIII), personaliza el diagnóstico con base en lo intelectual, lo emocional y lo somático de las manifestaciones (condición de la individualización efectiva del tratamiento). Utiliza los remedios a manera de influjos de energía sutil, elaborados a base de plantas, productos animales y muchas otras sustancias, que a partir de una «tintura madre», hace diluciones y dinamizaciones (agitación) hasta concentraciones infinitesimales. Elige los remedios más acordes con el diagnóstico, buscando una movilización sistémica específica que fortalezca (estimule) las fuerzas endógenas que subyacen a las manifestaciones, y así lograr la curación (la enfermedad, forma reactiva y peculiar de expresión de una disarmonía).

- La hipnosis, que puede considerarse una variante de la psicoterapia - precedió al psicoanálisis-, guarda grandes semejanzas con el EP, con la diferencia de que sus efectos intensificados se observan solo en las personas susceptibles. Consiste en una movilización sistémica inducida por sugestiones de gran poder debido a que inciden directamente en el plano psicosocial inconsciente, lo que provoca rearreglos asociativos afectivos que derivan en cambios de comportamiento, frecuentemente dramáticos (incluidos diversos tipos de trastornos psíquicos y somáti$\cos )$, que con el reforzamiento de la autohipnosis, 
modifican las formas de ser y de proceder de las personas tratadas.

No dudo que lo previo pueda juzgarse, más allá de un sano escepticismo, de inverosímil o hasta oscurantista, pero resulta que la escasa ciencia que ha incursionado en el «terreno vedado de las MA», en especial la idea de energía vital que es central en muchas de ellas, intentando responder sobre su «realidad o fantasía", ha encontrado indicios innegables de los flujos energéticos y de sus conductos circulatorios ${ }^{33}$. Al describirlas, no pretendo su defensa a ultranza, sino mostrar que estos saberes ancestrales que han resistido la prueba del tiempo entrañan posibilidades y potencialidades de alivio para muchos problemas crónicos que nos aquejan, que han sido ocultadas, negadas o ignoradas por los medios que difunden "verdades a medias» en favor de la industria de «la salud», proveídas por una ciencia extraviada de su sentido crítico originario, que impone un pensamiento único sobre «el buen conocimiento digno de crédito" y permanece subordinada a los intereses de lucro sin límite, que han colapsado la civilización ${ }^{10}$.

\section{Epílogo}

Al exponer la teoría de la MS y la ME, se ha pretendido, entre otras cosas, reivindicar el papel de las ideas en el conocimiento (potencialmente esclarecedor), en un momento histórico donde impera el pensamiento empirista-reduccionista - que debe su predominio a su sintonía con la dominancia de los intereses de lucro sin límite-, que ha descalificado el pensamiento teórico (crítica propositiva en el universo de las ideas) como especulación fútil, empobreciendo el saber científico al reducirlo a la comprobación de hechos. Esta teoría, al visibilizar una polaridad esencial de la práctica médica supresora/estimulante, que ha permanecido en la sombra y lejos de inquietar a la ciencia reduccionista, abre un espacio de reflexión inédito que aspira a sembrar dudas y ser caldo de cultivo de líneas de investigación inusitadas.

La ME no es una fantasía delirante de quien esto escribe. Su sentido es la omnipresencia del EP y la permanencia, contra viento y marea provenientes de la industria de la salud, de las MA de larga data. El $E P$, evidencia incuestionable de la existencia de fuerzas curativas endógenas y reveladora de la factibilidad de movilizarlas por cuenta propia (autosugestión), también sugiere la posibilidad de estimularlas en forma deliberada como recurso sanador de cada persona. Esto supondría aprender hábitos de las tradiciones cuna de las MA: concentración, introspección, meditación, mentalización, visualización en la búsqueda del autoconocimiento, autodominio, serenidad, "sintonía cósmica» o sanación. Sin embargo, el momento histórico es adverso al florecimiento de tales hábitos, con ambientes degradados y degradantes por «la deidad dinero», donde imperan el individualismo, la codicia, el consumismo, la competitividad, la incertidumbre, la urgencia perenne y la desazón perpetua, y la población deposita «el progreso" en las tecnologías que la suplantan, atrofian, mediatizan y deshumanizan ${ }^{9}$.

Como se dijo antes, el predominio de la MS no obedece a sus bondades, sino a su sintonía con los intereses de lucro que gobiernan el planeta. De ahí que las MA, al representar un obstáculo al negocio de la salud, sean víctimas de ocultamiento, marginación y descalificación, con la complicidad de una ciencia sometida a esa poderosa industria y presa del reduccionismo mecanicista (epígrafe), que les impone requisitos de cientificidad que las desvirtúa y, lo más grave, las excluye como objetos de conocimiento dignos. Lo anterior explica la anquilosis, el estancamiento, la degradación o la trivialización de sus prácticas en muchos casos, que le resta credibilidad a sus bondades y posibilidades, les impide ahondar en sus potencialidades o explorar nuevas en la búsqueda de contribuir al bien vivir de las personas ${ }^{6}$, y las hace más vulnerables a la simulación, el fraude o la estafa que permean todos los resquicios sociales.

Concluyo precisando que no se trata de desplazar la MS por la ME - algo imposible e inconveniente-, sino de proyectar la mirada teórica que incite el rescate de las MA para la indagación pertinente y la crítica constructiva; alentar investigaciones creativas capaces de superar la interpretación reduccionista imperante para adentrarse en la esfera psicosocial y sus interacciones con lo biológico y lo fisicoquímico; profundizar en las posibilidades y alcances de las MA; atreverse a idear y poner a prueba nuevas modalidades de ME promisorias, y así disponer de más elementos para ponderar las ventajas o desventajas de la ME respecto a la MS, a fin de definir prioridades, complementos, sinergias 0 reemplazos, para dotar a la población de mejores recursos en su aspiración del «bien vivir» más que de «Sobrevivir a toda costa» 6 , favoreciendo su autonomía y autogestión hacia formas de vida digna, mesurada, gratificante, serena y solidaria, a contrapelo del «aprisionamiento» de la medicalización. 


\section{Conflicto de intereses}

\section{El autor declara no tener ningún conflicto de intereses.}

\section{Bibliografía}

1. Viniegra-Velázquez L. La historia cultural de la enfermedad. Rev Invest Clin. 2008:60:527-44

2. Canguilhem G. Las concepciones de R. Leriche. Lo normal y lo patológico. México: Siglo XXI; 1978. pp. 63-71.

3. Viniegra-Velázquez L. El orden cultural, la enfermedad y el cuidado de la salud. Bol Med Hosp Infant Mex. 2017;74:397-406.

4. Leavell HR, Clark EG. Preventive medicine for the doctor in his community. An epidemiologic approach. New York: McGraw Hill; 1953.

5. Martínez González C, Riaño Galán I, Sánchez Jacob M. Prevención cuaternaria. La contención como imperativo ético. An Pediatr (Barc). 2014;81:396.e1-396.e8.

6. Viniegra-Velázquez L. El bien vivir: ¿cuidado de la salud o proyecto vital? Parte I. Bol Med Hosp Infant Mex. 2016:73:139-46.

7. Gérvas J, Pérez Fernández M. Uso y abuso del poder médico para definir enfermedad y factor de riesgo en relación con la prevención cuaternaria en España. Gac Sanit 2006;20(Supl. 1):127-34

8. Zackrisson S, Andersson I, Janzon L, Manjer J, Garne J. Rate of over-diagnosis of breast cancer 15 years after end of Malmö mammographic screening trial: follow up study. BMJ. 2006;332:689-92.

9. Viniegra-Velázquez L. La educación en nuestro tiempo: ¿competencia o aptitud? El caso de la medicina. Parte I. Bol Med Hosp Infant Mex. 2017;74:164-73

10. Viniegra-Velázquez L. El reduccionismo científico y el control de las conciencias. Parte II. Bol Med Hosp Infant Mex. 2014;71:323-8.

11. Ernst E. A systematic review of systematics reviews of homeopathy. $\mathrm{Br}$ J Clin Pharmacol. 2002;54:577-82.

12. National Health and Medical Research Council. 2015. NHMRC Information Paper: Evidence of effectiveness of homeopathy for treating health conditions. Canberra: National Health and Research Council; 2015.

13. Viniegra Velázquez L. El efecto placebo. Su dimensión teórica y sus implicaciones prácticas. Ciencia. 1987;38:131-46.

14. Beecher HK. Pain in men wounded in battle. Ann Surg. 1946;123:96-104

15. Beecher HK. Measurement of subjective responses. Quantitative effects of drugs. New York: Oxford University Press; 1959.
16. Porter JM, Culter BS, Lee BY, Reich T. Pentoxifylline efficacy in the treatment of intermittent claudication multicenter controlled double blind trial with objective assessment of chronic occlusive arterial disease patients. Am Heart J. 1982;104:66-74.

17. Morison RA, Woodmansey A, Young AJ. Placebo responses in arthritis trial. Ann Rheum Dis. 1961;20:179-84.

18. Amsterdam EA. New aspects of placebo response in angina pectoris. Am J Cardiol. 1969;20:249-54.

19. Budoff PW. Zomepirac sodium in the treatment of primary dysmenorrhea syndrome. N Engl J Med. 1982;307;714-17.

20. Molling PA, Lockner AW, Sauls RJ, Eisenberg L. Committed delinquent boys: the impact of perphenazine and of placebo. Arch Gen Psychiatry. 1962;7:70-4.

21. Wayne EJ. Placebos. Br Med J. 1956;2:157-62.

22. Leslie A. Ethics and practice of placebo therapy. Am J Med. 1954;16:854-7.

23. De Maria A, Craven DE, Heffernan JJ, McIntosh TK, Grindlinger GA Naloxone versus placebo in treatment of septic shock. Lancet. 1985;325:1363-5.

24. Palmer RS. The hypotensive action of Rauwolfia serpentina and reserpine: a double hidden placebo study of ambulatory patients with hypertension. Am Pract Digest Treat. 1955;6:1323-6.

25. Abbot FK, Mack M, Wolf $S$. The action of banthine on the stomach and duodenum of man with observations on the effects of placebos. Gastroenterol. 1952;20:249-54.

26. Katz HM, Bissel G. Blood sugar lowering effects of chloropropamide and tolbutamide. A double blind cooperative study. Diabetes. 1965;14: 650-4.

27. Zubieta JK, Stohler CS. Neurobiological mechanisms of placebo responses. Ann N Y Acad Sci. 2009;1156:198-210.

28. Peciña M, Zubieta JK. Molecular mechanisms of placebo responses in humans. Mol Psychiatry. 2015;20:416-23.

29. Viniegra-Velázquez L. Anticipación y ontogenia. Penetrando el proceso vital: más allá de la adaptación, el azar y la selección natural. Teoría de la interiorización del entorno y la anticipación. México: Ed. del autor; 2012. pp. 341-64

30. Ader R, Cohen N. Behaviorally conditioned immune suppression. Psychos Med. 1975;37:333-6.

31. Woods SC, Kulkosky PJ. Classically conditioned changes of blood glucose level. Psychos Med. 1976;38:201-5.

32. Honderich T, editor. Reductionism. The Oxford Companion of Philosophy. New York: Oxford University Press; 2005. pp.793-5.

33. Goldberg B, compilador. Medicina alternativa. La guía definitiva. California: Future Med Publish Inc; 1999. pp. 37-481. 